\title{
Drones, an air panopticon?
}

\section{SIGRADI2018 TECHNOPOLITICAS \\ xxii congresso da sociedade iberoamericana de gráfica digital 22th conference of the iberoamerican society of digital graphics $07|08| 09 \mid$ novembro|2018 iau usp | são carlos | sp br}

\author{
Diego Pimentel \\ Universidad Nacional de las Artes| Argentina | diegopimentel@gmail.com \\ Mariano Cataldi \\ Universidad Nacional de las Artes| Argentina | mariano7.3k@gmail.com \\ Gonzalo Muñiz \\ Universidad Nacional de las Artes| Argentina | contacto.gonzalomuniz@gmail.com
}

Néstor Barbitta

Universidad Nacional de las Artes| Argentina | nestorbarbitta@gmail.com

\begin{abstract}
Drones are not just a new technology or a fun toy airplane with remote control, they are also a possible new and silent control mechanism. It would not be the first time in history nor the last in which a technological advance is used to monitor society and impress upon it the power of the State. The particularity of this case is that not only could it be misused by the government, but also some members of society could come together in an anarchic movement that, for example, could invade people's privacy, as the fact of knowing the lack of control over this type of practices.
\end{abstract}

Keywords: Drones, VANT, intimidad, privacidad, seguridad.

\section{INTRODUCCIÓN}

"Tradicionalmente el poder es lo que se ve, lo que se muestra, lo que se manifiesta, y, de manera paradójica, encuentra el principio de su fuerza en el movimiento por el cual la despliega. [...] En cuanto al poder disciplinario, se ejerce haciéndose invisible; en cambio, impone a aquellos a quienes somete un principio de visibilidad obligatorio. En la disciplina, son los sometidos los que tienen que ser vistos. Su iluminación garantiza el dominio del poder que se ejerce sobre ellos. El hecho de ser visto sin cesar, de poder ser visto constantemente, es lo que mantiene en su sometimiento al individuo disciplinario." (Foucault, 1975)

La popularización de los vehículos aéreos no tripulados, VANT, (del inglés UAV: unmanned aerial vehicle), comúnmente conocidos como dron, no podría ser más oportuna para quienes ejercen el poder: en esta época donde prácticamente todo es posible de geolocalizar, de vigilar, controlar, medir y registrar, estas aves a control remoto representan, tal vez, una potencial amenaza a la privacidad del individuo.

Según una nota en Fortune (Vanian, 2017) para el año 2021 habrá alrededor de 3,6 millones de drones comerciales volando en el aire estadounidense, lo que representa el triple de drones con respecto a 2016, mientras que en el Reino Unido, según el Departamento de Transporte del Gobierno Británico, todos los drones por encima de 250 gramos, deberán ser registrados.

Por otro lado, Google, una de las empresas que almacena la mayor cantidad de datos privados del mundo, le está ofreciendo recursos al Departamento de Defensa de los Estados Unidos en el Proyecto Maven (2017), utilizando inteligencia artificial en el análisis de datos recopilados por drones, el big data y el deep learning en zonas de guerra para tener una clara ventaja sobre el enemigo.

\section{Metodología}

La investigación tuvo como punto de partida el estudio de casos de tecnología disruptiva, analizando su impacto cultural, social y político (internet, los dispositivos con conectividad, las redes sociales, etc), ahondando a la vez en temas como:

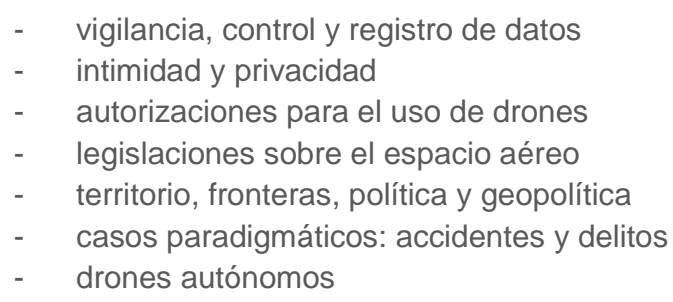

1. Relevamiento de distintos vehículos aéreos no tripulados y sistemas de reconocimiento facial, como drones comerciales y software tanto privado como de código abierto.

2. Ciudades y lugares donde se ha implementado el concepto de panóptico a través de sus cámaras, drones y sistemas de reconocimiento facial para la vigilancia de sus ciudadanos. 
3. Distintos puntos de vista sobre cómo estos sistemas de vigilancia afectan la vida de las personas.

\section{ESTADO ACTUAL DEL CONOCIMIENTO}

Sin responder a una necesidad concreta, como puede ser la de los dispositivos móviles, los drones son cada vez más parte de la vida cotidiana del entretenimiento doméstico.

Según una nota publicada en el sitio web del BBVA (2016), los drones comerciales avanzan en la conquista del mercado, en parte, gracias al precio cada vez más accesible de los mismos, producto del interés de las compañías y de los consumidores. La publicación afirma que para fines de aquel año los envíos de drones a consumidores en todo el mundo superaría los 7,3 millones y alcanzaría los 29 millones en 2021.

Dos años después, ese pronóstico parecería estar en lo cierto. El aumento de la venta de drones para uso doméstico no se detiene y su curva asciende conforme crece su popularidad.

Lo cierto es que, independientemente de la exactitud del pronóstico, nos enfrentamos ante una realidad que invita a realizar algunas preguntas:

¿Qué consecuencias podría acarrear el uso de drones domésticos en grandes proporciones por parte de la sociedad?

En 1954 se estrena La ventana indiscreta (Rear Window) dirigida por Alfred Hitchcock y basada en el relato de Cornell Woolrich It Had to Be Murder. En la película, el actor principal -un fotógrafo que se ve obligado a permanecer en reposo con una pierna enyesada producto de un accidente- intenta huir del hastío que tal inmovilización le produce mediante el uso de binoculares observando, desde la ventana de su departamento, lo que ocurre en las viviendas de enfrente. Debido a una serie de extrañas circunstancias empieza a sospechar de un vecino cuya mujer ha desaparecido.

Sesenta y cuatro años después veríamos absurdo el uso de binoculares como objeto y método para la investigación de este crimen contando con las múltiples posibilidades que ofrece un dron, por ejemplo el Phantom 4 de la empresa china DJI: alcance aproximado de vuelo de hasta $8 \mathrm{~km}$ de su control remoto, cámara de video $4 \mathrm{k}$ y capacidad de alcanzar los 2000 metros de altura. Es decir, desde el sillón de nuestra casa podemos ver lo que ocurre en 80 cuadras a la redonda en una resolución que cuadruplica la definición de la mayoría de los televisores hogareños en Argentina.

\section{MARCO TEÓRICO}

Jeremy Bentham (1791), filósofo, economista, pensador y escritor inglés, padre del utilitarismo, elabora un modelo de cárcel al que denomina Panóptico. Esta prisión, cuyo nombre proviene de la raíz griega "verlo todo" (pan- opticón), se basaba en una construcción circular con una torre de vigilancia en el centro desde donde se podría vigilar a los presos desde todo punto de vista sin que éstos pudieran advertir si en la torre había efectivamente alguien o no.

El objetivo de crear un sentimiento de omnisciencia invisible sobre los detenidos tenía por fin instalar sobre los detenidos tenía por fin instalar sobre los aquellos un sentimiento de interiorización hasta el punto de vigilarse a sí mismos.

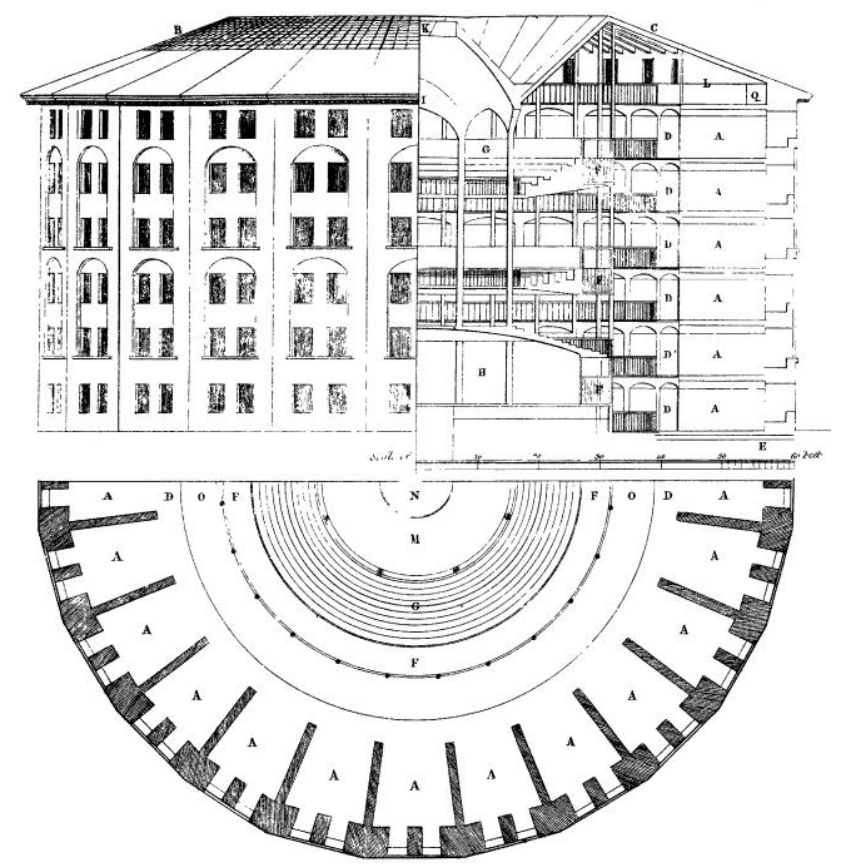

Figura 1. Diseño del Panóptico de Bentham. The works of Jeremy Bentham vol. IV, 172-3.

En Vigilar y castigar, Michel Foucault (1975) toma el concepto de Panóptico como ejemplo perfecto del ejercicio de la vigilancia: provocar en el prisionero una conciencia permanente, casi paranoica, de un poder que lo vigila constantemente, un ojo que todo lo ve, como sucede en "1984", la novela distópica escrita por George Orwell (1949). Es decir, garantizar el funcionamiento automático del poder, independientemente de que se esté ejerciendo o no.

Como sostiene Deleuze (1999), se trata de controlar: "Todos los centros de encierro atraviesan una crisis generalizada: cárcel, hospital, fábrica, escuela, familia. (...) Los ministros competentes anuncian constantemente las supuestamente necesarias reformas. Reformar la escuela, reformar la industria, reformar el hospital, el ejército, la cárcel; pero todos saben que, a un plazo más o menos largo, estas instituciones están acabadas. [...] Solamente se pretende gestionar su agonía y mantener a la gente ocupada mientras se instalan esas nuevas fuerzas que ya están llamando a nuestras puertas. Se trata de las sociedades de control, que están sustituyendo a las disciplinarias. "Control" es el nombre propuesto por Burroughs para designar al nuevo monstruo que Foucault reconoció como nuestro futuro inmediato. (...) En las 
sociedades disciplinarias siempre había que volver a empezar (terminada la escuela, empieza el cuartel, después de éste viene la fábrica), mientras que en las sociedades de control nunca se termina nada: la empresa, la formación o el servicio son los estados metaestables y coexistentes de una misma modulación, una especie de deformador universal."

227 años después de la idea del panóptico de Bentham, su principal objetivo se vuelve una realidad: China está creando "la red de videovigilancia más grande y más sofisticada del mundo" (figura 2). La misma cuenta con 170 millones de cámaras distribuidas por todo el país para controlar a sus más de 1.300 millones de personas. La mayoría de estas cámaras poseen tecnología de reconocimiento facial, de texto y objetos. De esta manera el gobierno puede verificar la foto de un transeúnte con la de su documento de identidad, accediendo a toda su información y pudiendo rastrear sus movimientos. La policia facilmente puede encontrar a cualquier persona en 7 minutos o menos. Yin Jun, vicepresidente de Investigación y Desarrollo de Dahua Technology afirma en una entrevista con la BBC que "podemos relacionar tu rostro con tu carro, con tus familiares y con las personas con las que estuviste en contacto" (BBC Mundo, diciembre 2017). La idea de privacidad se pone en jaque. Organizaciones de derechos humanos como Humans Rights Watchs aseguran que es una violación a la privacidad" y apunta a "seguir y predecir las actividades de los disidentes". Según el poeta crítico del gobierno, Ji Feng, "puedes sentir los ojos puestos en ti todos los días".

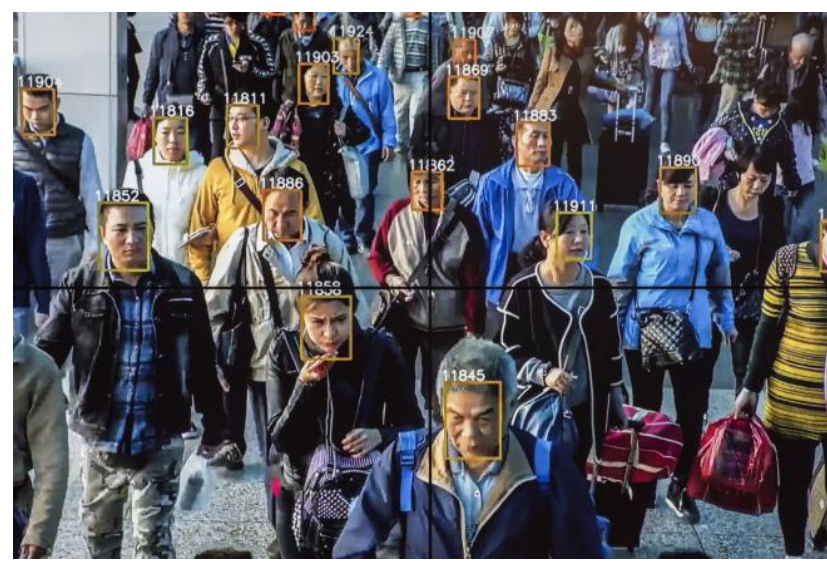

Figura 2: Monitor de vigilancia utilizando el sistema de reconocimiento facial en Beijing. (Gilles Sabrié / The Washington Post)

Por otro lado, Prosegur (2015), una empresa de seguridad española, ha lanzado un dron con capacidad autónoma de vigilancia que propone una evolución de los servicios tradicionales de seguridad. Este dispositivo funciona guiado por un algoritmo de posicionamiento que se basa en distintos sensores de altura y en un escáner láser, reconociendo su entorno y con la capacidad de detectar obstáculos y resolver rutas para superarlos. El equipo cuenta con una cámara de video común y otra térmica o infrarroja que le permiten detectar la temperatura corporal de personas y otros seres vivos.

\section{DRONES AUTÓNOMOS}

Un dron autónomo es aquel que cuenta con algoritmos que le ofrecen diferentes posibilidades de acción frente a una decisión a tomar, siendo el dron quien finalmente elige cuál de todas esas alternativas es la mejor.

Este tipo de tecnología ya está siendo probada por varias universidades e instituciones de investigación militar y es posible encontrar en internet videos con demostraciones de "arrays de drones" moviéndose como enjambres, formando imágenes aéreas, desplazándose e interactuando con total autonomía.

La pregunta sobre qué consecuencias traería aparejadas el hackeo de miles de drones militares por parte de grupos terroristas es inevitable. Incluso basta con que sean únicamente hackers para pensar en consecuencias fatales.

El 3 de mayo de 2018, en California, Estados Unidos, a las 8.31 PM, 958 drones iluminados crearon en el aire la que sería la portada de la edición del 11 de junio de la célebre revista Time, titulada "The Dron Age. A Special Report" (figura 3). Si bien estos drones recibieron órdenes humanas para la representación aérea de la tapa, no se registró ningún accidente entre los casi mil drones al momento de volar hasta llegar cada uno a su puesto. Para la consecución de la idea, Time trabajó junto a Intel's Drone Light Show team, Astraeus Aerial Cinema Systems y L.A. Drones.

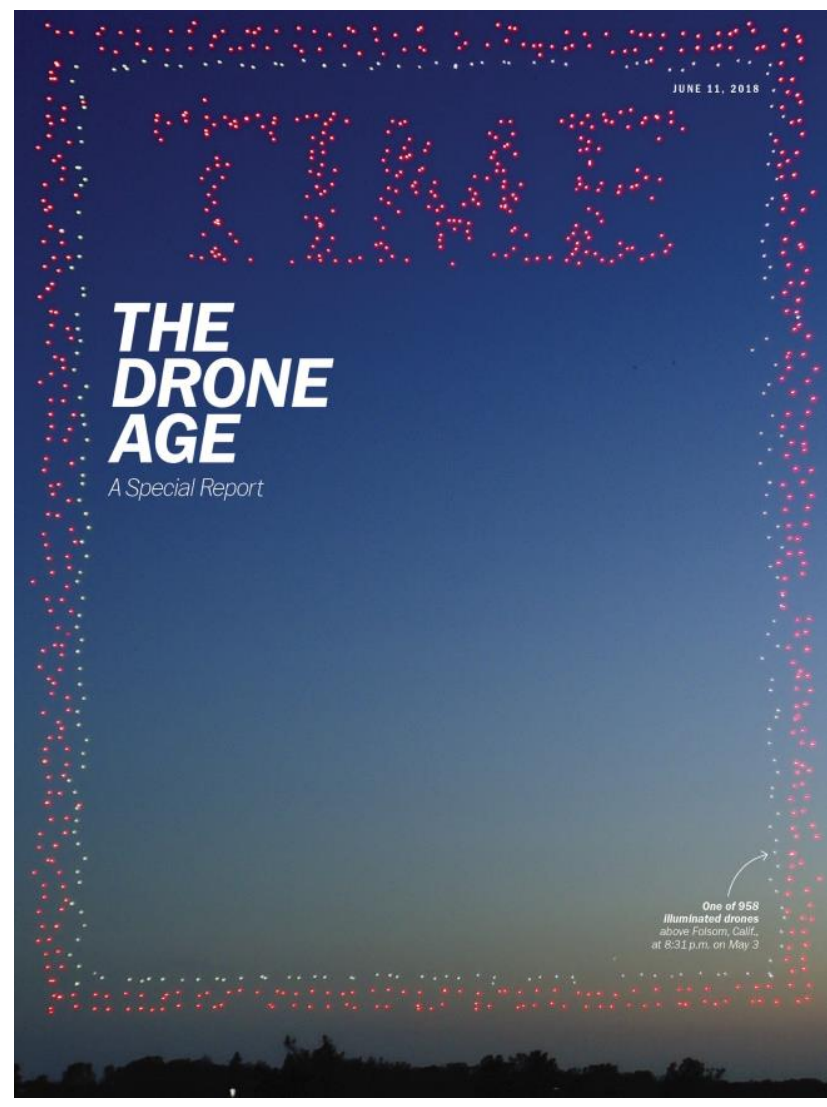

Figura 3 - Imagen del icónico logo de la revista Time y su borde rojo realizada utilizando 958 drones de la compañía norteamericana INTEL en el espacio aéreo de California. E.E.U.U. Mayo 2018. 


\section{OBJETIVOS E HIPÓTESIS DE INVESTIGACIÓN}

La hipótesis que rige esta investigación radica en la proyección de un futuro distópico planteando dos escenarios posibles: la utilización de drones por parte de los Estados para ejercer la vigilancia, el control, el poder y los dueños de los drones domésticos ejerciendo un uso anárquico.

¿Qué pasaría si el Estado decidiera llenar el espacio aéreo con drones para registrar lo que sucede en cada una de las manifestaciones? ¿Aquellos manifestantes serían indiferentes a tal situación? ¿Podríamos pensar en un Estado que se sirve del Big Data para guardar y cruzar información en contra del individuo, amedrentándolo con el gran ojo que todo lo controla y todo lo sabe?

Por otro lado, ¿qué pasaría si algunos de los millones de dueños de drones domésticos decidieran jugar a ser los guardias de un panóptico desde la invisibilidad que estos objetos les confieren? Imaginemos por un instante vernos en nuestro sillón favorito del living y advertir, de pronto, una pequeña luz roja flotando en el aire. Nos están filmando. Han perpetrado nuestra intimidad, nuestra privacidad. ¿Hace cuánto? ¿Qué han visto? ¿Lo subirá a Internet? ¿Se viralizará en las redes sociales? "Odio Nacional", el capítulo de la serie Black Mirror, está más cerca de la realidad que de la ficción.

Surgieron en consecuencia los siguientes interrogantes:
1) ¿Existe una regulación sobre privacidad y espacio aéreo para casos como este?

2) Más allá de su existencia, ¿cómo se impediría?

3) En caso de perpetrarse un ataque de estas características, ¿es factible determinar quién manipuló el dron que cometió el acto ilícito?

Los drones de uso civil tienen restringido por GPS la zonas de vuelo. En Estados Unidos existen zonas de vuelo restringidas que se pueden desbloquear a través de la web del ente regulador aeronáutico. Existen zonas llamadas No Fly Zone que no permiten ningún tipo de desbloqueo debido a la sensibilidad de la zona. (Aeropuertos, Bases Militares, Edificios Gubernamentales, etc.). Sin embargo estas regulaciones no agotan la problemática anteriormente planteada.

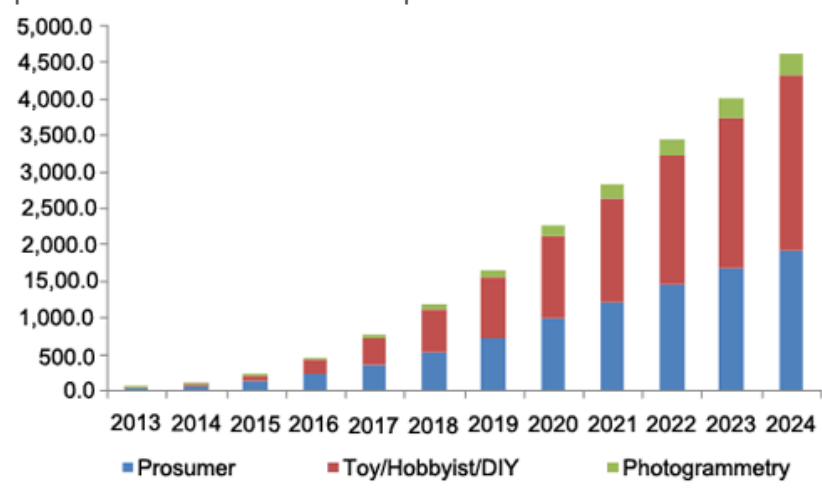

Figura 4. Compradores de drones en el mercado de Estados Unidos, según tecnología. Proyecciones 2013-2024. (en Millones de dólares)

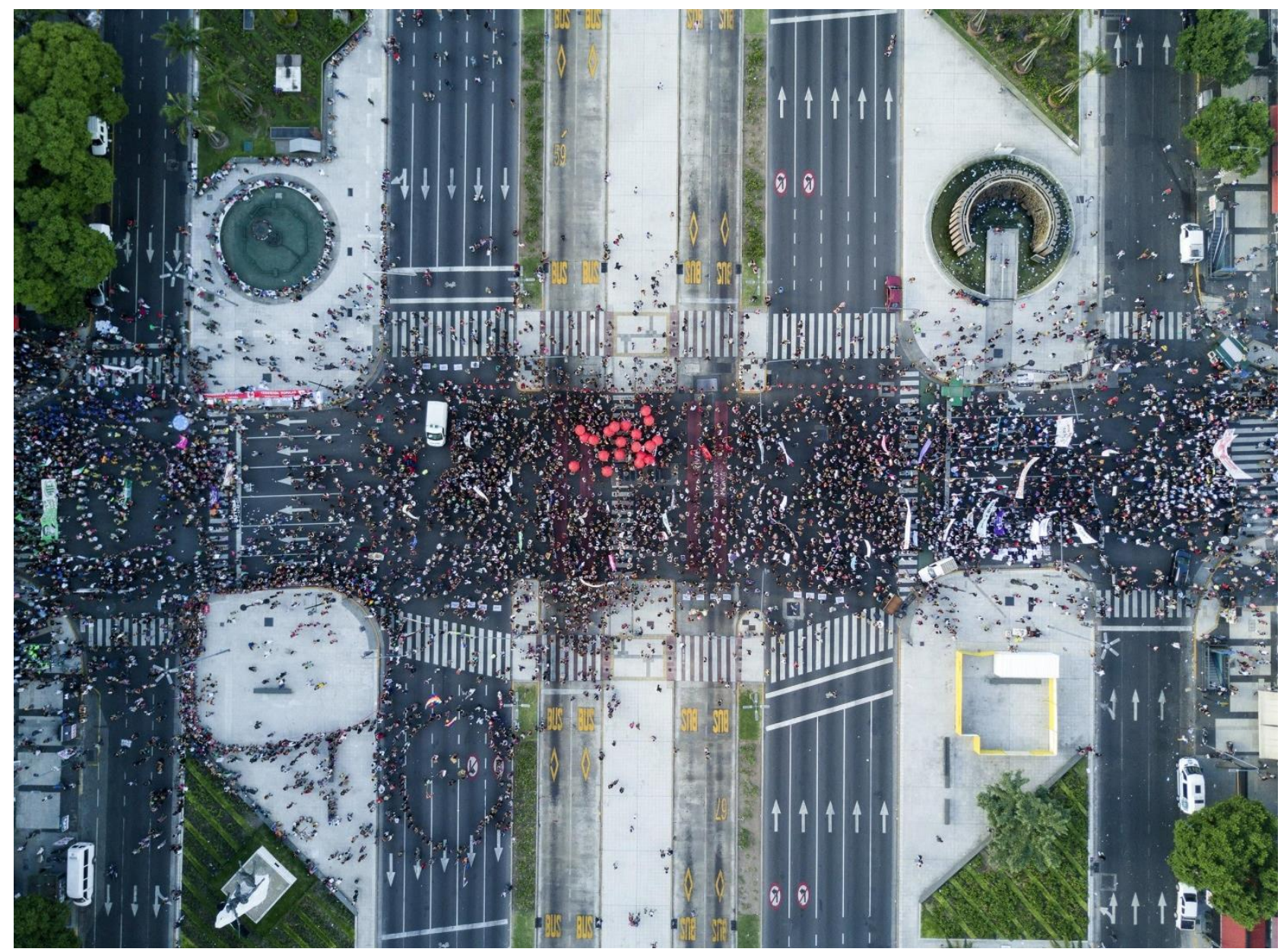

Figura 5: Fotografía digital, toma directa realizada con drone DJI Mavic Pro. Marcha del 8M (Día Internacional de la Mujer), Ciudad de Buenos Aires. Altura: 111 mts. Autor: Néstor Barbitta, 2017. 


\section{Resultados}

Establecer un registro y un esquema conceptual que permita:

- Registrar y mapear los antecedentes de la utilización de drones como herramientas de control por parte de los Estados.

- Definir el alcance que puedan llegar a tener los drones, la inteligencia artificial, el big data y la deep learning, en relación a nuestra seguridad y libertad de acción.

- Las implicancias de tener objetos voladores como parte de nuestra cotidianeidad, desde logistica, transporte y vigilancia, y los efectos que pueden tener en el medio ambiente y seguridad.

\section{Discusión}

El desarrollo de la hipótesis planteada a propósito de las consecuencias de la libre comercialización y utilización de drones es la contribución de este proyecto, advirtiendo sobre los posibles riesgos $y / 0$ peligros en torno a la libertad y la seguridad de la sociedad. Un interrogante sobre el futuro cercano del avance de los drones sobre la propiedad privada y el espacio público.

\section{REFERENCIAS}

Bentham, J., (2002), Le Panoptique. París, Francia: Editorial Mille et une nuits.

Deleuze, G., (2014) El Poder. Curso sobre Foucault. Buenos Aires, Argentina: Editorial Cactus

Deleuze, G., (1999) Conversaciones. Post-scriptum sobre las sociedades del control, Valencia, España: Editorial PreTextos.

Foucault, M., (2008), La arqueología del saber, Buenos Aires, Argentina: Editorial Siglo XXI Editores.

Foucault, M., (2008), Un diálogo sobre el poder y otras conversaciones, Buenos Aires, Argentina: Editorial Alianza.

Foucault, M., (2000), Las redes del poder, Buenos Aires, Argentina: Editorial Prometeo Libros.

Foucault, M., (1976), Vigilar y castigar. Nacimiento de la prisión, México DF, México: Editorial Siglo XXI.

Fromm, E., (1993) El miedo a la libertad, Barcelona, España: Editorial Paidós.

Nietzsche, F., (2000) La voluntad de poder, Madrid, España: Editorial Edaf.

Orwell, G., (2013) 1984, Buenos Aires, Argentina: Editorial Debolsillo.

Sartre, J. P., (2009), El existencialismo es un humanismo, Barcelona, España: Editorial Edhasa.

Pellerin, C., (2017). Project Maven to Deploy Computer Algorithms to War Zone by Year's End. Retrieved from:
https://www.defense.gov/News/Article/Article/1254719/projectmaven-to-deploy-computer-algorithms-to-war-zone-by-yearsend/

Departamento de Transporte del Gobierno Británico (2017) Drones to be registered and users to sit safety tests under new government rules. Retrieved from:

https://www.gov.uk/government/news/drones-to-be-registeredand-users-to-sit-safety-tests-under-new-government-rules

BBVA. (2016) ¿Quién lidera el mercado de los drones?. Retrieved from:

https://www.bbva.com/es/quien-lidera-mercado-drones/

Vanian, J., (2017). Drone Use Will Skyrocket By 2021, Government Says. Retrieved from:

http://fortune.com/2017/03/22/federal-government-droneskyrocket/

BBC Mundo (2017) China, el Estado que todo lo ve: así es la red de videovigilancia más grande y más sofisticada del mundo. Retrieved from:

http://www.bbc.com/mundo/noticias-internacional-42398920

Hernández Bonilla, J. M., (2018) Reconocimiento facial, el panóptico universal y contemporáneo. Retrieved from:

https://www.elespectador.com/tecnologia/reconocimiento-facial-elpanoptico-universal-y-contemporaneo-articulo-744412

Gordon, R. (2017) CSAIL team's system of quadcopters that fly and drive suggest another approach to developing flying cars. Retrieved from:

http://news.mit.edu/2017/drones-drive-flying-cars-0626

Prosegur España (2015) Prosegur presenta el primer dron de vigilancia capaz de volar autónomamente en interiores. Retrieved from:

https://www.prosegur.es/newsdetails/drone-vigilancia-interiores

NATO Review Magazine (2017) Autonomous military drones: no longer science fiction. Retrieved from:

https://www.nato.int/docu/review/2017/also-in-2017/autonomousmilitary-drones-no-longer-science-fiction/EN/index.htm 